\title{
Modulated Model Predictive Control for Induction Motor Drives with Sequential Cost Function Evaluation
}

\author{
Valerio Vodola \\ Dept. of Energy \\ Politecnico di Torino \\ Turin, Italy \\ valerio.vodola@gmail.com \\ Margarita Norambuena \\ Dept. of Electrical Engineering \\ Valparaiso, Chile \\ margarita.norambuena@usm.cl \\ Jose Rodriguez \\ Dept. of Engineering Science \\ Universidad Andres Bello \\ Santiago, Chile \\ jose.rodriguez@unab.cl
}

Universidad Tecnica Federico Santa Maria

\author{
Shafiq Odhano \\ Dept. of Electrical and Electronic Engineering \\ University of Nottingham \\ Nottingham, UK \\ shafiq.odhano@nottingham.ac.uk
}

\author{
Cristian Garcia \\ Dept. of Electrical Engineering \\ Universidad de Talca \\ Curico, Chile \\ cgarciap7@gmail.com
}

\author{
Silvio Vaschetto \\ Dept. of Energy \\ Politecnico di Torino \\ Turin, Italy \\ silvio.vaschetto@polito.it
}

\author{
Pericle Zanchetta \\ Dept. of Electrical and Electronic Engineering \\ University of Nottingham \\ Nottingham, UK \\ pericle.zanchetta@nottingham.ac.uk
}

\author{
Radu Bojo \\ Dept. of Energy \\ Politecnico di Torino \\ Turin, Italy \\ radu.bojoi@polito.it
}

\begin{abstract}
Sequential model predictive control is a recent innovation in the high-performance control of electric drives. The elimination of weighting factors and associated tuning work is among the biggest advantages of this MPC implementation. The cost function evaluation takes place in two steps with each step narrowing down the choice of optimal voltage vector to be applied at the next switching instant. Like the conventional finite control states MPC, the sequential MPC also has a disadvantage of variable switching frequency. In this paper, this problem is addressed by considering the sequential MPC implementation with a modulator. After two-step cost function evaluation, the optimal and second optimal voltage vectors' duty cycles are computed based on the slope of the controlled variables. This preserves the optimality of the solution while, at the same time, guaranteeing constant switching frequency and reduced current and torque ripples in the drive response.

Index Terms-Model predictive control, variable speed drives, predictive torque control.
\end{abstract}

\section{INTRODUCTION}

Field Oriented Control (FOC) and Direct Torque Control (DTC) are the most commonly used control strategies in variable frequency ac motor drives [1]-[3]. In recent years, thanks to the improved microcontroller performance, more sophisticated control strategies have been developed. These new strategies include the Finite Control Set Model Predictive Control (FCS-MPC) of torque and flux that uses a single cost function in which the flux and torque errors are considered at the same time. To give more or less importance to the two objectives, a weight is used [4], [5]. The calculation of this weight is often obtained through iterative processes that are not simple and above all not accepted by all users [6], [7].

To solve these problems, a new control strategy called sequential MPC (SMPC) has been recently proposed [8]. In SMPC control strategy, two cost functions are defined separately: one for the torque and one for the stator flux, which are then evaluated sequentially. In this control strategy, a two-level Voltage Source Inverter (2L-VSI) is used to supply the induction motor. The 2L-VSI can generate seven different voltage vectors, in particular six active voltage vectors and one zero vector. In the SMPC strategy of [8], the torque cost function is evaluated for all seven voltage vectors available, then the two voltage vectors that generate the smallest torque error are selected for the evaluation of the flux error. At the end, from the two voltage vectors available from torque optimization step, the voltage vector that minimize the stator flux error is selected and it is used to supply the machine. So, with this control strategy all the problems related to the definition of the weights are avoided.

Whenever an MPC technique is used, the presence of a non-constant switching frequency causes a high harmonic spectrum, and in addition the absence of amplitude modulation of the voltage vectors, causes a high current ripple and consequently a poor torque quality. To solve the problems mentioned above, the modulation to a (2L-VSI) was implemented. The results obtained by introducing two modulation techniques for 
a new control strategy called SMPC-FT3 will be presented in this paper. SMPC-FT3 is a strategy that exploits the concepts of sequential evaluation of the cost functions proposed in [8], but in which the evaluation of the cost functions is carried out in reverse way. In particular the SMPC-FT3 is a strategy that in the first step evaluates the flux cost function for all the seven voltage vectors available, then the three voltage vectors that generate the smallest flux error are selected for the evaluation of the torque error. At the end, from the three voltage vectors available, the voltage vector that minimizes the torque error is selected.

To apply the two vectors modulation to this new control strategy, the global optimum deriving from the torque error minimization is modulated with the zero vector, while in the case of the three vectors modulation, in addition to the global optimum and the zero vector also the second optimum is used. The modulation has also been implemented for the SMPC strategy [8].

This paper introduces the concept and demonstrates that including a modulator brings the SMPC at par with its competitor control strategies while keeping the advantages of no weighting factor tuning requirement. Additionally, simple and straightforward duty cycle computation formula are presented for two-vector and three-vector modulation. Simulation and experimental result are presented to validate the proposed control strategy.

\section{MAthematical MOdel OF THE INVERTER AND THE INDUCTION MACHINE}

The simulated inverter is the 2-level Voltage Source Inverter (2L-VSI) with ideal switches and with a DC-link of $520 \mathrm{~V}$. As regards the inverter circuit and the vectors that can be generated, it is possible to refer to [9]. The dynamic equations of IM in stationary frame $(\alpha, \beta)$ are:

$$
\begin{aligned}
\mathbf{v}_{s} & =R_{s} \mathbf{i}_{s}+\frac{d \lambda_{s}}{d t}, \\
0 & =R_{r} \mathbf{i}_{r}+\frac{d \lambda_{r}}{d t}-j \omega_{r} \lambda_{r}, \\
\lambda_{s} & =L_{s} \mathbf{i}_{s}+L_{m} \mathbf{i}_{r}, \\
\lambda_{r} & =L_{r} \mathbf{i}_{r}+L_{m} \mathbf{i}_{s}, \\
T & =1.5 p\left(\lambda_{s} \wedge \mathbf{i}_{s}\right), \\
J_{m} \frac{d \omega}{d t} & =T-T_{L},
\end{aligned}
$$

where $\mathbf{v}_{s}$ is the stator-voltage, $R_{s}=0.41 \Omega$ and $R_{r}=0.31 \Omega$ are the stator and rotor resistance, $\lambda_{s}$ and $\lambda_{r}$ are the stator and rotor flux, $\mathbf{i}_{s}$ and $\mathbf{i}_{r}$ are the stator and rotor current, $\omega_{r}$ and $\omega$ are the electrical and mechanical rotor speed, $L_{s}=0.09757 \mathrm{H}$, $L_{r}=0.09757 \mathrm{H}$ and $L_{m}=0.09187 \mathrm{H}$ are the stator the rotor and the mutual inductance, $T$ and $T_{L}$ are the motor and load torque, respectively, $p=2$ is the number of pole-pairs and $J_{m}$ $=0.062 \mathrm{kgm}^{2}$ is the moment of inertia of the machine. It is important to note that bold typeface is used for vector quantities and " $\mathrm{j}$ " is a complex operator.

\section{THE CONTROL STRATEGY SMPC-FT3}

For the implementation of SMPC-FT3, a stator flux $\lambda_{s}$ and stator current $\mathbf{i}_{s}$ must be predicted, then from these two quantities it is possible to predict the torque that will be supplied by the machine. It is important to compensate the digital delay [10], this mean that $\lambda_{s}, \mathbf{i}_{s}$ and $T$ must be calculated for the future instant $k+2$. For the implementation of this strategy a flux observer [11] was used, from it for every instant, exploiting the properties of the discrete integral, two information are available and they are:

- $\lambda_{s}^{k+1}$ which is a predicted stator flux for the future instant $k+1$, in particular it is the output of the back-emf integration at the present sample $k$.

- $\lambda_{s}^{k}$ which is the stator flux at the previous instant $k$, kept in memory.

The first information of the flux observer is used for the prediction of the current and stator flux at the instant $k+2$, while the second information is just used for the prediction of the stator current at the instant $k+1$. For prediction of stator current the current state equation is:

$$
\begin{aligned}
\frac{d \mathbf{i}_{s}}{d t}= & \frac{1}{\sigma L_{s}}\left(\mathbf{v}_{s}-\left(R_{s}+R_{r} \frac{L_{s}}{L_{r}}\right) \mathbf{i}_{s}+j \omega_{r} \sigma L_{s} \mathbf{i}_{s}\right. \\
& \left.+\frac{\lambda_{s}}{\tau_{r}}-j \omega_{r} \lambda_{s}\right),
\end{aligned}
$$

where $\sigma=\left(1-L_{m}^{2} /\left(\left(L_{r} L_{s}\right)\right)\right.$ is the leakage factor and $\tau_{r}=L_{r} R_{r}$ is the rotor time constant. The above equation can be obtained starting from (1) and substituting (3) and (4). Using the Euler discretization and on the basis of the measured current, of the voltage vector applied at the instant $k$ and $\lambda_{s}^{k}$, it is possible to calculate the current at the instant $k+1$ as below:

$$
\begin{aligned}
\mathbf{i}_{s}^{k+1}= & \mathbf{i}_{s}^{k}+\frac{T_{s}}{\sigma L_{s}}\left(\mathbf{v}_{s}^{k}-\left(R_{s}+R_{r} \frac{L_{s}}{L_{r}}\right) \mathbf{i}_{s}^{k}+j \omega_{r} \sigma L_{s} \mathbf{i}_{s}^{k}\right. \\
& \left.+\frac{\lambda_{s}^{k}}{\tau_{r}}-j \omega_{r} \lambda_{s}^{k}\right) .
\end{aligned}
$$

In the same way it is possible to obtain $\mathbf{i}_{s}^{k+2}$, using the results of (8) and the flux observed at the current time $\mathrm{k}$ that is $\lambda_{s}^{k+1}$. The stator flux prediction at the instant $k+2$ is obtained by the forward Euler discretization of (1), using the stator current $\mathbf{i}_{s}^{k+1}$ and considering $T_{s}$ as the sampling time.

$$
\lambda_{s}^{k+2}=\lambda_{s}^{k+1}+T_{s} \mathbf{v}_{s}^{k+1}-R_{s} T_{s} \mathbf{i}_{s}^{k+1} .
$$

From the prediction of the current and flux at instant $k+2$, it is possible to calculate the torque, at instant $k+2\left(T^{k+2}\right)$, using (5). The two cost functions used for evaluating torque and flux error are:

$$
\begin{aligned}
& g_{\lambda}=\left(\left|\lambda_{s}^{*}\right|-\left|\lambda_{s}^{k+2}\right|\right)^{2}, \\
& g_{T}=\left(T^{*}-T^{k+2}\right)^{2},
\end{aligned}
$$

where $\left|\lambda_{s}^{*}\right|$ is the reference stator flux amplitude, $\left|\lambda_{s}^{k+2}\right|$ is the predicted stator flux modulus at the instant $k+2, T^{*}$ is the reference torque exiting from speed PI regulator. 


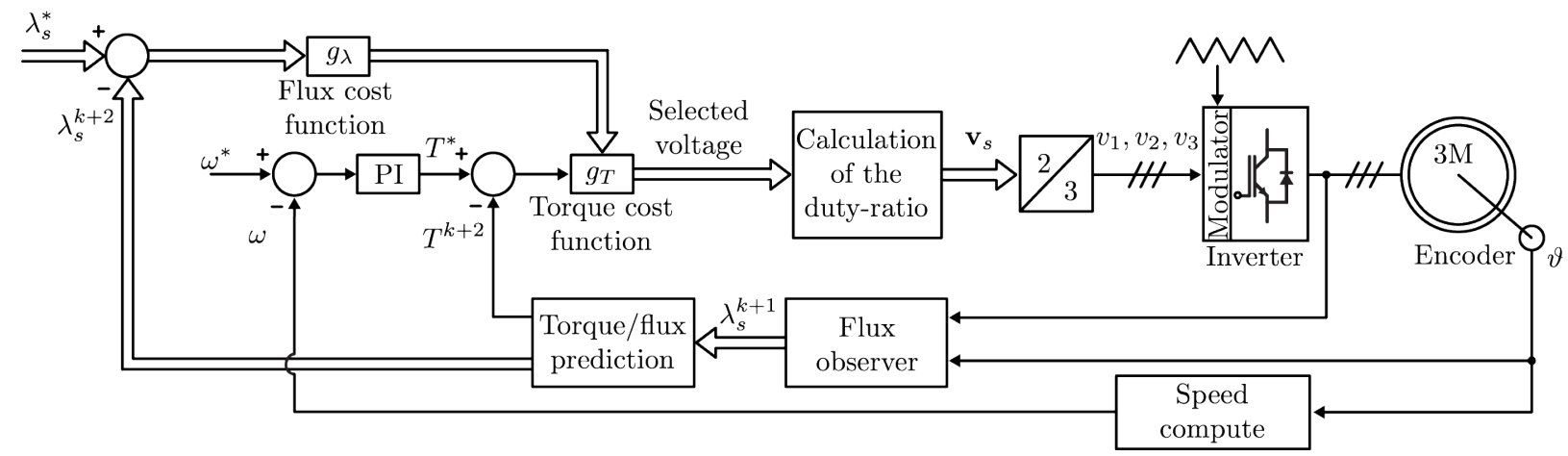

Fig. 1: Block diagram of the SMPC-FT3.

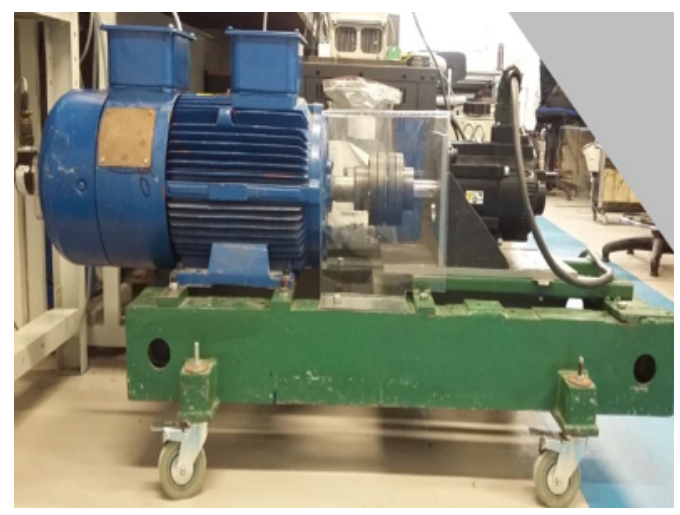

Fig. 2: Test bench used for experimental analyses control.

\section{SMPC-FT3 WITH MODULATION THAT USE TWO VOLTAGE VECTORS}

Fig. 1 shows the block diagram of the SMPC-FT3 control strategy, while Fig. 2 shows the test bench to be used for experimental analyses. Unlike [8], the control strategy studied here begins with the evaluation of $g_{\lambda}$ for all seven available voltage vectors; among these vectors the three that produce the smallest flux error are chosen for the evaluation of $g_{T}$. At the end the chosen vector is the one that minimizes the torque error $g_{T}$. If this vector is the zero vector $\left(v_{0}\right)$, a zero voltage vector is applied to the machine, if it is non-zero voltage vector $\left(v_{n 0}\right)$, it will be modulated in amplitude using a coefficient that is called duty-ratio $(d)$. The duty-ratio is calculated so as to be able to have, within a control period, the torque delivered by the machine equal to the desired torque considering in any case the digital delay compensation. To do this, it is necessary to calculate the torque derivative in the case in which the nonzero vector is applied, without amplitude modulation, $\left(s^{v_{n 0}}\right)$, and in case the zero vector is applied $\left(s^{v_{0}}\right)$. The relationships used to calculate $\left(s^{v_{n 0}}\right)$ are exactly the same as those used for the calculation of $\left(s^{v_{0}}\right)$, therefore only the main relations used for the calculation of $\left(s^{v_{n 0}}\right)$ will be shown. $s^{v_{n 0}}$ can be calculated as shown in (12) using (11).

$$
s^{v_{n 0}}=\frac{3}{2} p\left(\frac{d \lambda_{s, v_{n 0}}^{k+2}}{d t} \wedge \mathbf{i}_{s, v_{n 0}}^{k+2}-\frac{d \mathbf{i}_{s, v_{n 0}}^{k+2}}{d t} \wedge \lambda_{s, v_{n 0}}^{k+2}\right),
$$

with $d \lambda_{s, v_{n 0}}^{k+2} / d t, d \mathbf{i}_{s, v_{n 0}}^{k+2} / d t, \lambda_{s, v_{n 0}}^{k+2}$ and $\mathbf{i}_{s, v_{n 0}}^{k+2}$ are respectively the flux and current derivatives, and the values of flux and current evaluated at time $k+2$ in the case $v_{n 0}$ is applied. In particular $d \lambda_{s, v_{n 0}}^{k+2} / d t$ can be obtained from (1), and calculated as (13), then from (13) using the backward Euler discretization it is also possible to obtain $\lambda_{s, v_{n 0}}^{k+2}$, as shown in (14).

$$
\begin{aligned}
\frac{d \lambda_{s, v_{n 0}}^{k+2}}{d t} & =v_{n 0}^{k+1}-R_{s} \mathbf{i}_{s, v_{n 0}}^{k+1}, \\
\lambda_{s, v_{n 0}}^{k+2} & =\lambda_{s}^{k+1}+T_{s} v_{n 0}^{k+1}-R_{s} T_{s} \mathbf{i}_{s, v_{n 0}}^{k+1} .
\end{aligned}
$$

On the other hand, $d \mathbf{i}_{s, v_{n 0}}^{k+2} / d t$ can be obtained using (7) and calculated as in (15), then from (15) using the backward Euler discretization it is also possible to obtain $\mathbf{i}_{s, v_{n 0}}^{k+2}$, as shown in (16).

$$
\begin{aligned}
\frac{d \mathbf{i}_{s, v_{n 0}}^{k+2}}{d t}= & \frac{1}{\sigma L_{s}}\left(v_{n 0}^{k+2}-\left(R_{s}+R_{r} \frac{L_{s}}{L_{r}}\right) \mathbf{i}_{s}^{k+1}+j \omega_{r} \sigma L_{s} \mathbf{i}_{s}^{k+1}\right. \\
& \left.+\frac{\lambda_{s}^{k+1}}{\tau_{r}}-j \omega_{r} \lambda_{s}^{k+1}\right) \\
\mathbf{i}_{s, v_{n 0}}^{k+2}= & \mathbf{i}_{s}^{k+1}+\frac{T_{s}}{\sigma L_{s}}\left(v_{n 0}^{k+2}-\left(R_{s}+R_{r} \frac{L_{s}}{L_{r}}\right) \mathbf{i}_{s}^{k+1}\right. \\
& \left.+j \omega_{r} \sigma L_{s} \mathbf{i}_{s}^{k+1}+\frac{\lambda_{s}^{k+1}}{\tau_{r}}-j \omega_{r} \lambda_{s}^{k+1}\right) .
\end{aligned}
$$

Once $s^{v_{n 0}}$ and $s^{v_{0}}$ are calculated, and considering that the application of the $v_{0}$ causes a reduction of torque while the application of $v_{n 0}$ increases the torque, see Fig. 3, it is intuitive to understand that in order to have the torque error $\left(\varepsilon_{T}\right)$, within each control period, equal to zero (17) must be respected.

$$
\varepsilon_{T}=\left|E_{T}+s^{v_{n 0}} d T_{s}+s^{v_{0}}(1-d) T_{s}\right|=0,
$$

where $E_{T}$ is the torque error at the beginning of each control period, $T_{s}$ is the sampling time and $d$ is the duty-ratio. Finally, by solving (17) the value of duty ratio for the active vector can be calculated using (18).

$$
d=\frac{s^{0} T_{s}+E_{T}}{T_{s}\left(s^{n 0}-s^{0}\right)},
$$

with $d$ that must always be limited within $[0,1]$. Calculated the value of the duty-ratio, it is therefore possible to define the voltage amplitude in the fixed frame $(\alpha, \beta)$ and the voltage with which to supply the machine. 


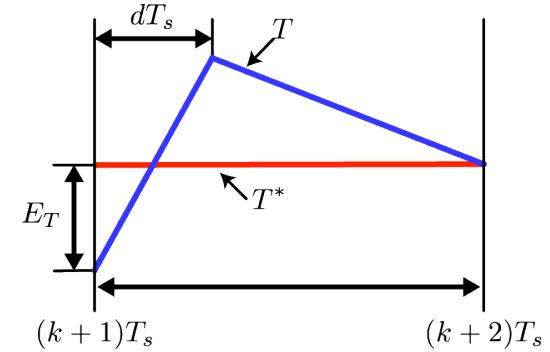

Fig. 3: Objective of the strategy at each control step and main quantities to be calculated.

TABLE I: Principals control parameters

\begin{tabular}{|c|cc|}
\hline Parameter & Value & Unit \\
\hline \hline Sample time $T_{s}$ & 40 & {$[\mu s]$} \\
DC-link voltage $V_{D C}$ & 520 & {$[\mathrm{~V}]$} \\
Bandwidth speed regulator $\omega_{b}$ & 314,16 & {$[\mathrm{rad} / \mathrm{s}]$} \\
Proportional gain of the speed regulator & $\omega_{b} \cdot J_{m}$ & \\
Integral gain of the speed regulator & $\omega_{b}^{2} \cdot J_{m}$ \\
\hline
\end{tabular}

\section{REsults}

Table I shows the principal control parameters, used to test the SMPC-FT3 control strategy without modulator with two vector modulations and with three vectors modulations. In all cases, the response to a speed step and a load step was tested. In particular, at 0.2 seconds a step speed reference $\left(\omega^{*}\right)$ was imposed equal to $100[\mathrm{rad} / \mathrm{s}]$, while at 0.6 seconds a load step of $40 \mathrm{Nm}$ was required. From 0 [s] to 0.2 [s] the machine is fluxing.

A. SMPC-FT3 with two vectors modulation and without modulation

In Fig. 4 and Fig. 5 the results obtained using the SMPC-FT3 strategy with two vector modulation and without modulator are shown. It is possible to affirm that from the dynamic point of view, there are not important differences, in fact the rise times of the speed are completely comparable as shown in Fig. 4(a) and Fig.5(a). Fig. 4(b) and Fig.5(b) it can be observed how, thanks to the introduction of modulation, the torque ripple is reduced and consequently also the currents become decidedly less noisy in any operating condition as shown by the Fig. 4(c) and Fig.5(c). Finally, it can be observed that despite the voltage vector selected by the torque error minimization is modulated in amplitude, it is possible to respect the flux reference imposed at $0.8 \mathrm{Vs}$.

A starting operation in the experimental setup for SMPCFT3 strategy with two vector modulation and without modulator are shown in Fig. 9 and Fig. 8, respectively. The speed response in similar in both cases, Fig. 9(a) and Fig. 8(a), with a typical second order response due to the PI controller. The torque performance is improve in the modulated case (Fig. 9(b)), presenting a ripple reduction compared to the SMPC-FT3 without modulation. However, the experimental result of SMPC-FT3 with two vector modulation has an offset that it is not observed in the simulation result of the same strategy, Fig. 4(b). This offset is attributed to experimental
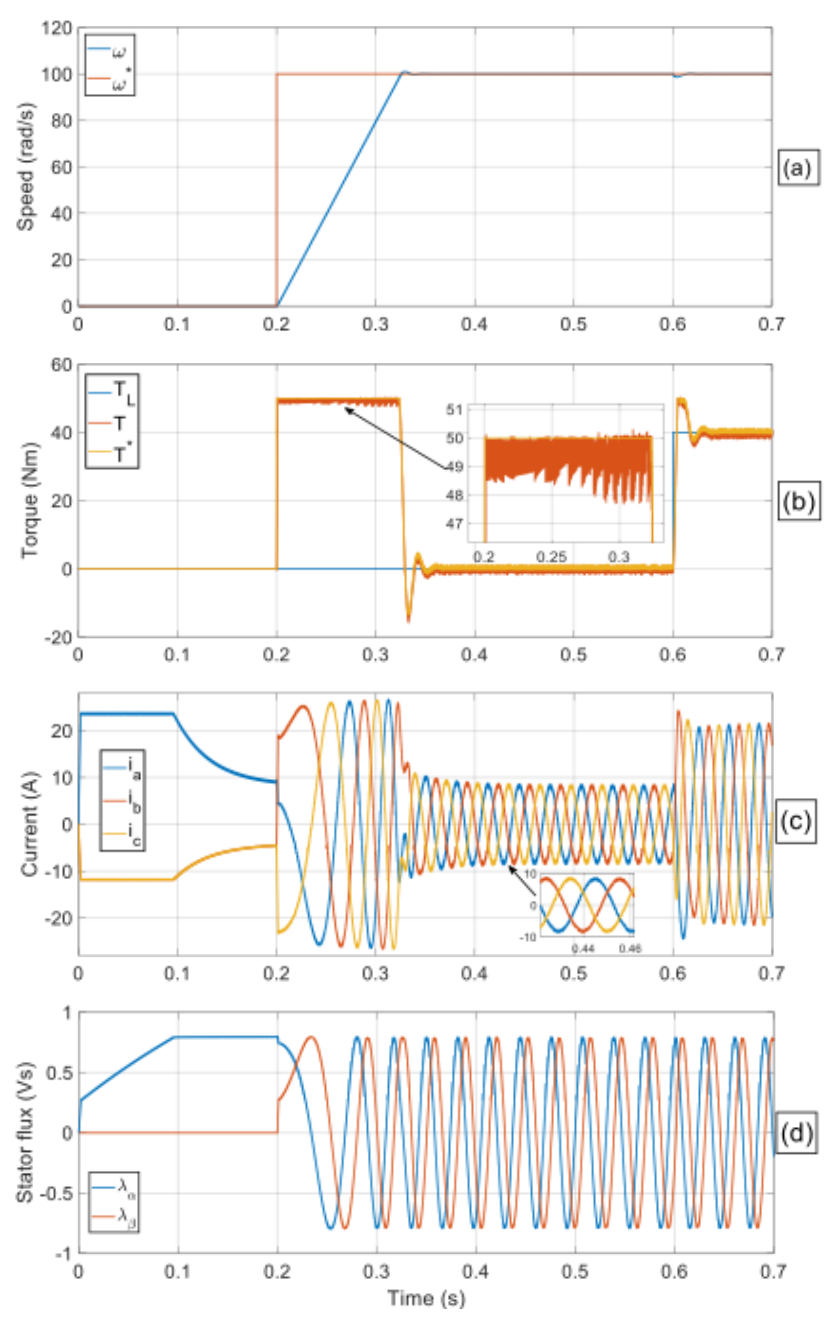

Fig. 4: SMPC-FT3 with two vector modulation: (a) Speed; (b) Torque; (c) Stator current; (d) Stator flux.

things not considered, as for example, the dead-time of the semiconductors, the difference in the real parameters, etc. And also because it is not possible to obtain exactly the desired reference with two vectors.

\section{B. SMPC-FT3 with three vectors modulation and without modulation}

With the aim of further improving the performance obtained with the SMPC-FT3 with two vectors modulations strategy, the modulation with three vectors was also introduced. In this modulation technique, in addition to use the zero voltage vector and the voltage vector that produce the smallest torque error, the voltage vector that produces the second smallest torque error is also used. Fig. 6 and Fig. 7 show respectively the torque and the currents obtained with the modulation technique which uses three voltage vectors. It can be observed that with this modulation technique it is possible to obtain a further improvement in terms of torque and current ripple.

Fig. 10 shows an experimental starting maneuver where SMPC-FT3 with three vector modulation is implemented. The 

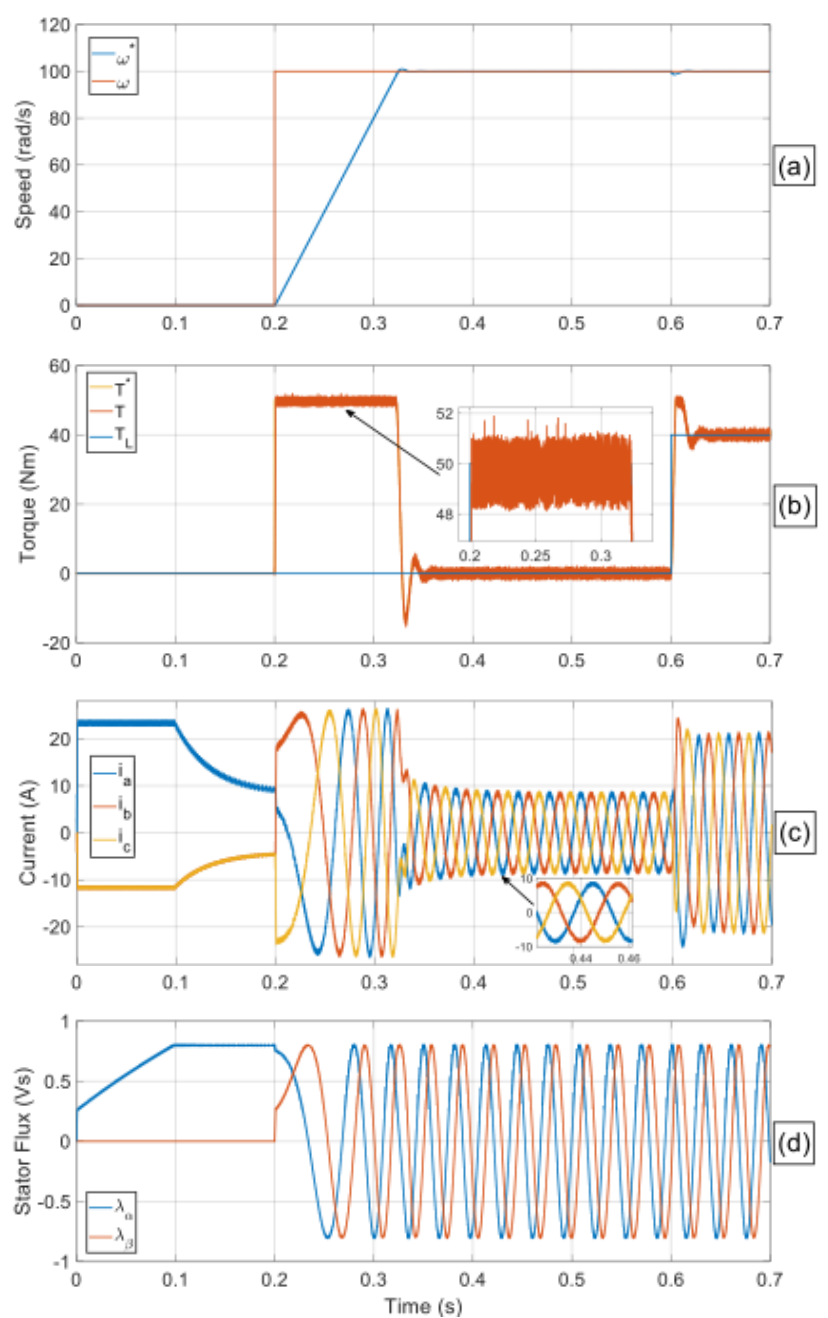

Fig. 5: SMPC-FT3 without modulation: (a) Speed; (b) Torque; (c) Stator current; (d) Stator flux.

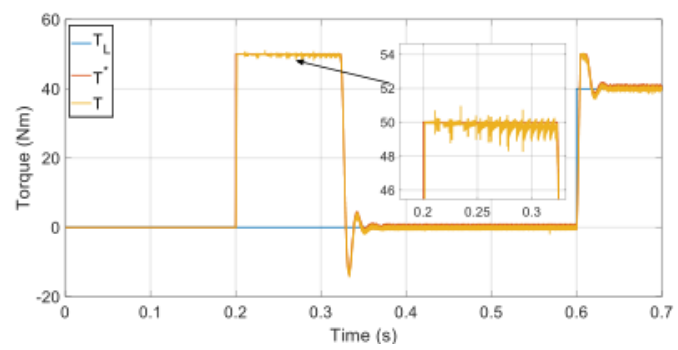

Fig. 6: SMPC-FT3 with three vector modulation: Torque delivered by the machine, reference torque and required load torque.

speed response, Fig. 10(a), is good and similar to the cases of SMPC-FT3 without modulation (Fig. 8(a)) and SMPC-FT3 with two vector modulation (Fig. 9(a)). The torque is shown in Fig. 10(b), the behavior does not have an evident offset as the case of SMPC-FT3 with two vectors modulations, this thanks to the fact that the three vectors allow to obtain exactly the

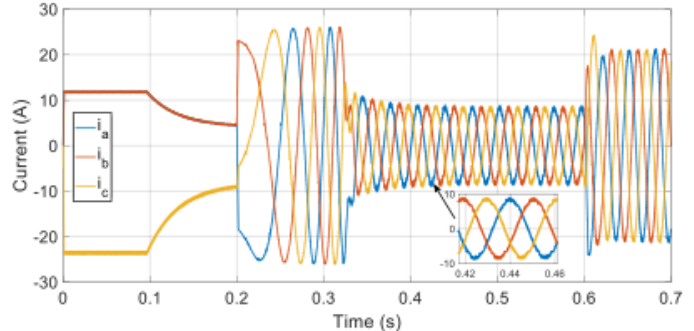

Fig. 7: SMPC-FT3 with three vector modulation phases current.
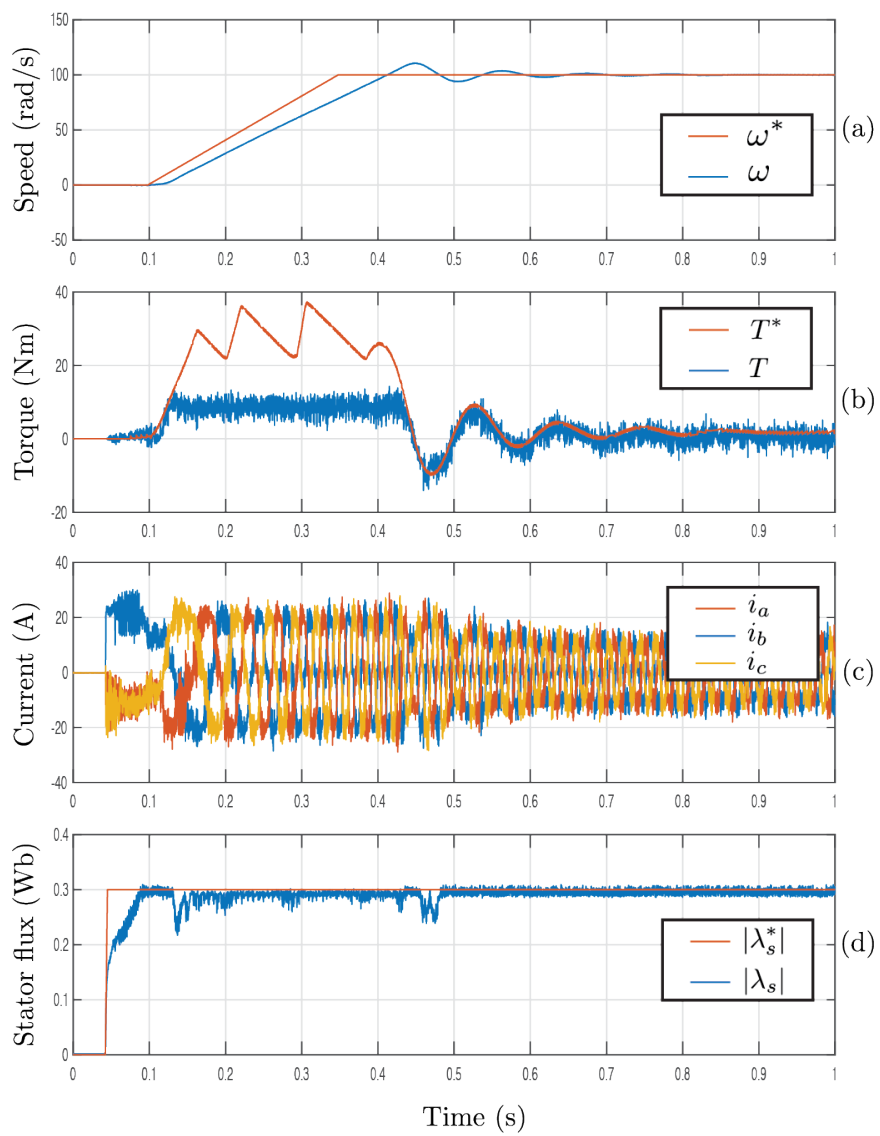

Fig. 8: Experimental SMPC-FT3 without modulation: (a) Speed; (b) Torque; (c) Stator current; (d) Stator flux.

reference.

\section{ACKNOWLEDGMENT}

The authors acknowledge in part the support of CONICYT/FONDECYT Initiation Research Project 11180235 and in part the support of CONICYT/FONDECYT Research Project 1170167.

\section{Conclusions}

This paper presents various possibilities of modulation applied to the SMPC-FT3 control of an induction motor drive. The SMPC-FT3 is based on a new sequential model predictive 

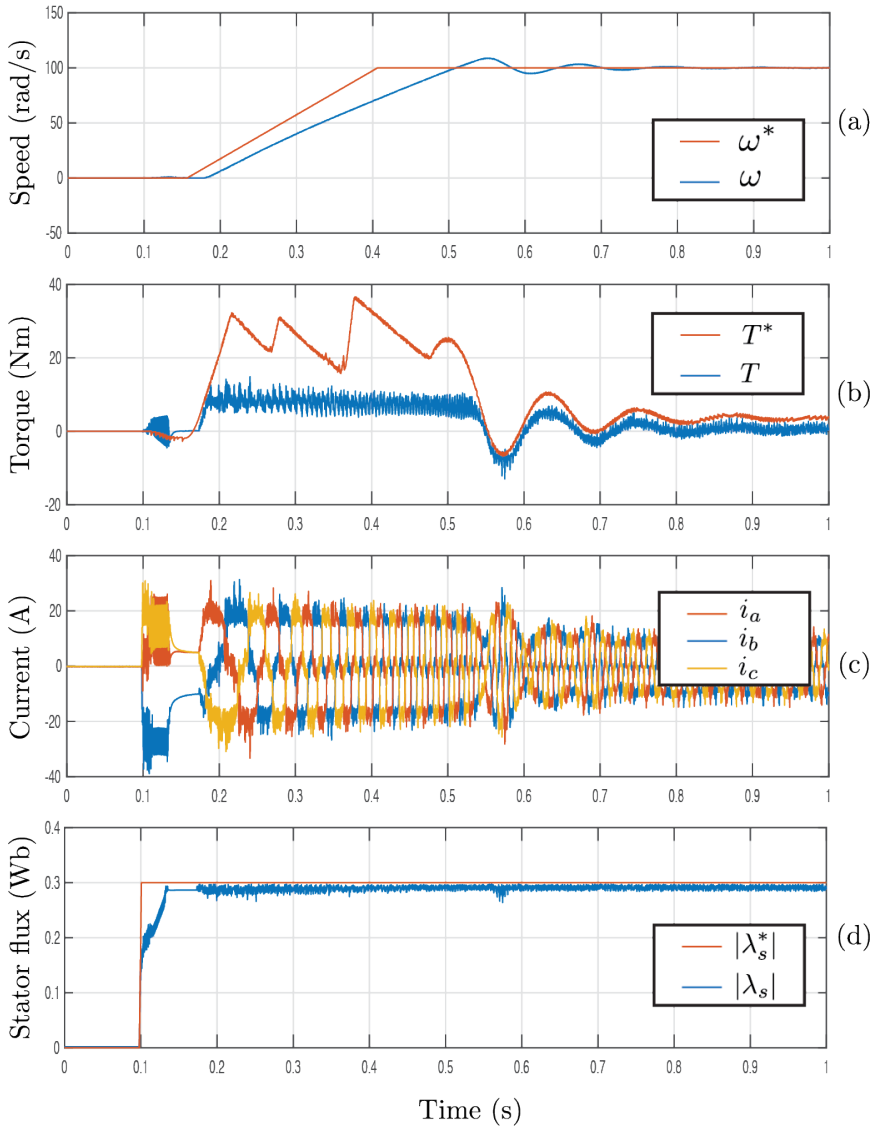

Fig. 9: Experimental SMPC-FT3 with two vector modulation: (a) Speed; (b) Torque; (c) Stator current; (d) Stator flux.

control strategy [8] that has been recently proposed for highperformance control of electric drives.

The simulation and experimental result demonstrated that SMPC-FT3 with modulation is a suitable strategy for electrical drives that keeps the simplicity of SMPC-FT3 without weighting factor and allows to obtain significant improvements in terms of torque quality and current ripple thanks to the inclusion of a modulation stage.

\section{REFERENCES}

[1] F. Blaschke, "The principle of field-orientation applied to the transvector closed-loop control system for rotating field machines," Siemens, p. Vol 3, 1972.

[2] R. Gabriel, W. Leonhard, and C. J. Nordby, "Field-oriented control of a standard ac motor using microprocessors," IEEE Transactions on Industry Applications, vol. IA-16, no. 2, pp. 186-192, March 1980.

[3] G. S. Buja and M. P. Kazmierkowski, "Direct torque control of pwm inverter-fed ac motors - a survey," IEEE Transactions on Industrial Electronics, vol. 51, no. 4, pp. 744-757, Aug 2004.

[4] S. A. Davari, D. A. Khaburi, and R. Kennel, "An improved fcsmpc algorithm for an induction motor with an imposed optimized weighting factor," IEEE Transactions on Power Electronics, vol. 27, no. 3, pp. 1540-1551, March 2012.

[5] M. Norambuena, S. Dieckerhoff, S. Kouro, and J. Rodriguez, "Finite control set model predictive control of a stacked multicell converter with reduced computational cost," in IECON 2015 - 41st Annual Conference of the IEEE Industrial Electronics Society, Nov 2015, pp. 001819 001824
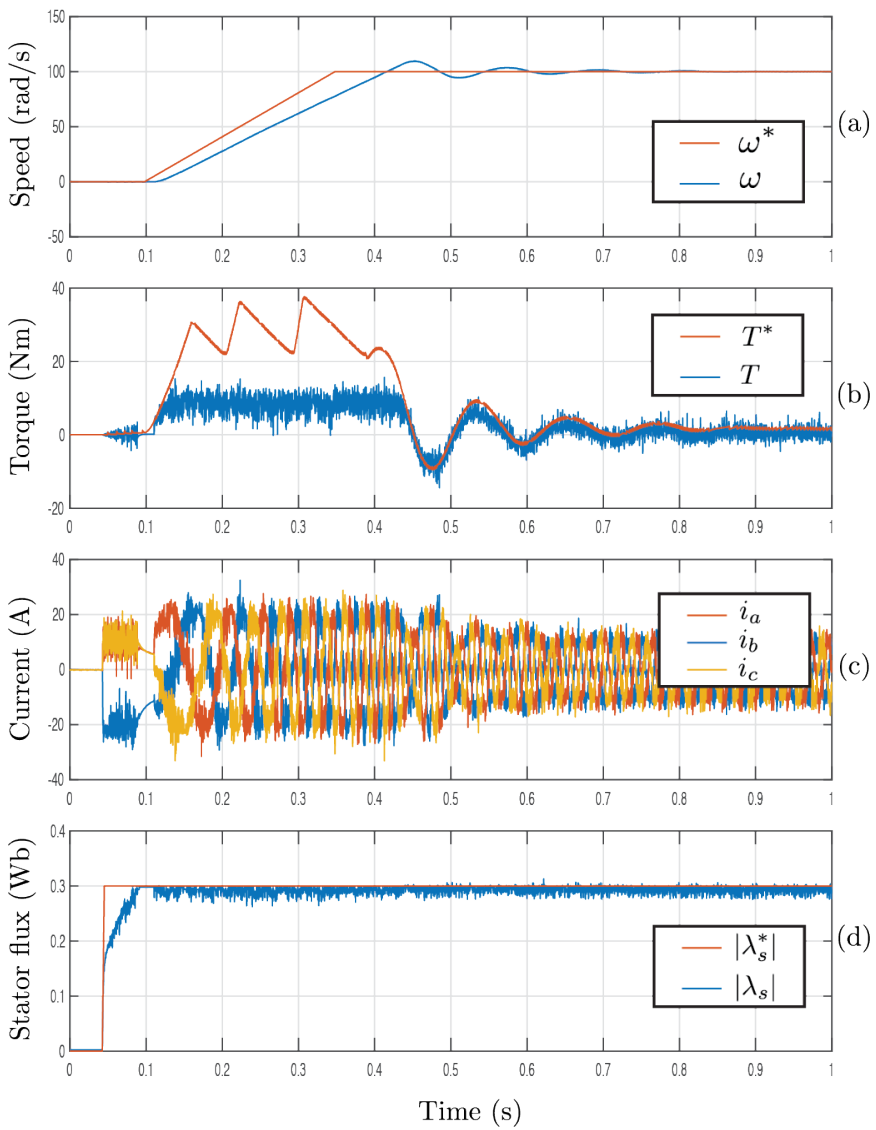

Fig. 10: Experimental SMPC-FT3 with three vector modulation: (a) Speed; (b) Torque; (c) Stator current; (d) Stator flux.

[6] Y. Zhang and H. Yang, "Two-vector-based model predictive torque control without weighting factors for induction motor drives," IEEE Transactions on Power Electronics, vol. 31, no. 2, pp. 1381-1390, Feb 2016.

[7] Z. Zhang, Wei Tian, Wanyi Xiong, and R. Kennel, "Predictive torque control of induction machines fed by 31-npc converters with online weighting factor adjustment using fuzzy logic," in 2017 IEEE Transportation Electrification Conference and Expo (ITEC), June 2017, pp. $84-89$.

[8] M. Norambuena, J. Rodriguez, Z. Zhang, F. Wang, C. Garcia, and R. Kennel, "A very simple strategy for high-quality performance of ac machines using model predictive control," IEEE Transactions on Power Electronics, vol. 34, no. 1, pp. 794-800, Jan 2019.

[9] C. F. Garcia, C. A. Silva, J. R. Rodriguez, P. Zanchetta, and S. A. Odhano, "Modulated model-predictive control with optimized overmodulation," IEEE Journal of Emerging and Selected Topics in Power Electronics, vol. 7, no. 1, pp. 404-413, March 2019.

[10] P. Cortes, J. Rodriguez, C. Silva, and A. Flores, "Delay compensation in model predictive current control of a three-phase inverter," IEEE Transactions on Industrial Electronics, vol. 59, no. 2, pp. 1323-1325, Feb 2012.

[11] S. A. Odhano, A. Boglietti, R. Bojoi, and E. Armando, "Unified direct-flux vector control of induction motor self-commissioning drive with analysis of parameter detuning effects," in 2013 IEEE Energy Conversion Congress and Exposition, Sep. 2013, pp. 2071-2078. 